Second International Workshop on

Functional and Operatorial Statistics.

Santander, June 16-18, 2011

\title{
Functional kernel estimators of conditional extreme quantiles
}

\author{
Laurent GARDES** and Stéphane GIRARD* \\ Team Mistis, INRIA Rhône-Alpes and LJK, Inovallée, 655, av. de l'Europe, \\ Montbonnot, 38334 Saint-Ismier cedex, France. \\ * Stephane.Girard@inrialpes.fr
}

\begin{abstract}
We address the estimation of "extreme" conditional quantiles i.e. when their order converges to one as the sample size increases. Conditions on the rate of convergence of their order to one are provided to obtain asymptotically Gaussian distributed kernel estimators. A Weissman-type estimator and kernel estimators of the conditional tailindex are derived, permitting to estimate extreme conditional quantiles of arbitrary order.
\end{abstract}

\section{Introduction}

Let $\left(X_{i}, Y_{i}\right), i=1, \ldots, n$ be independent copies of a random pair $(X, Y)$ in $E \times \mathbb{R}$ where $E$ is a metric space associated to a distance $d$. We address the problem of estimating $q\left(\alpha_{n} \mid x\right) \in \mathbb{R}$ verifying $\mathbb{P}\left(Y>q\left(\alpha_{n} \mid x\right) \mid X=x\right)=\alpha_{n}$ where $\alpha_{n} \rightarrow 0$ as $n \rightarrow \infty$ and $x \in E$. In such a case, $q\left(\alpha_{n} \mid x\right)$ is referred to as an extreme conditional quantile in contrast to classical conditional quantiles (known as regression quantiles) for which $\alpha_{n}=\alpha$ is fixed in $(0,1)$. While the nonparametric estimation of ordinary regression quantiles has been extensively studied, see for instance the seminal papers (Roussas, 1969), (Stone, 1977) or (Ferraty and Vieu, 2006, Chapter 5) less attention has been paid to extreme conditional quantiles despite their potential interest. Here, we focus on the setting where the conditional distribution of $Y$ given $X=x$ has an infinite endpoint and is heavy-tailed, an analytical characterization of this property being given in the next section. We show, under mild conditions, that extreme conditional quantiles $q\left(\alpha_{n} \mid x\right)$ can still be estimated through a functional kernel estimator of $\mathbb{P}(Y>. \mid x)$. We provide sufficient conditions on the rate of convergence of $\alpha_{n}$ to 0 so that our estimator is asymptotically Gaussian 
distributed. Making use of this, some functional kernel estimators of the conditional tail-index are introduced and a Weissman type estimator (Weissman, 1978) is derived, permitting to estimate extreme conditional quantiles $q\left(\beta_{n} \mid x\right)$ where $\beta_{n} \rightarrow 0$ arbitrarily fast.

\section{Notations and assumptions}

The conditional survival function (csf) of $Y$ given $X=x$ is denoted by $\bar{F}(y \mid x)=$ $\mathbb{P}(Y>y \mid X=x)$. The kernel estimator of $\bar{F}(y \mid x)$ is defined for all $(x, y) \in E \times \mathbb{R}$ by

$$
\hat{\bar{F}}_{n}(y \mid x)=\sum_{i=1}^{n} K\left(d\left(x, X_{i}\right) / h\right) Q\left(\left(Y_{i}-y\right) / \lambda\right) / \sum_{i=1}^{n} K\left(d\left(x, X_{i}\right) / h\right),
$$

with $Q(t)=\int_{-\infty}^{t} q(s) d s$ where $K: \mathbb{R}^{+} \rightarrow \mathbb{R}^{+}$and $q: \mathbb{R} \rightarrow \mathbb{R}^{+}$are two kernel functions, and $h=h_{n}$ and $\lambda=\lambda_{n}$ are two nonrandom sequences such that $h \rightarrow 0$ as $n \rightarrow \infty$. In this context, $h$ and $\lambda$ are called window-width. This estimator was considered for instance in (Ferraty and Vieu, 2006, page 56). In Theorem 1, the asymptotic distribution of (1) is established when estimating small tail probabilities, i.e when $y=y_{n}$ goes to infinity with the sample size $n$. Similarly, the kernel estimators of conditional quantiles $q(\alpha \mid x)$ are defined via the generalized inverse of $\hat{\overline{F_{n}}}(. \mid x)$ :

$$
\hat{q}_{n}(\alpha \mid x)=\inf \left\{t, \hat{\bar{F}}_{n}(t \mid x) \leq \alpha\right\},
$$

for all $\alpha \in(0,1)$. Many authors are interested in this type of estimator for fixed $\alpha \in(0,1)$ : weak and strong consistency are proved respectively in (Stone, 1977) and (Gannoun, 1990), asymptotic normality being established when $E$ is finite dimensional by (Stute, 1986), (Samanta, 1989), (Berlinet et al., 2001) and by (Ferraty et al., 2005) when $E$ is a general metric space. In Theorem 2, the asymptotic distribution of (2) is investigated when estimating extreme quantiles, i.e when $\alpha=\alpha_{n}$ goes to 0 as the sample size $n$ goes to infinity. The asymptotic behavior of such estimators depends on the nature of the conditional distribution tail. In this paper, we focus on heavy tails. More specifically, we assume that the csf satisfies

$$
\text { (A1): } \bar{F}(y \mid x)=c(x) \exp \left\{-\int_{1}^{y}\left(\frac{1}{\gamma(x)}-\varepsilon(u \mid x)\right) \frac{d u}{u}\right\}
$$

where $\gamma($.$) is a positive function of the covariate x, c($.$) is a positive function and |\varepsilon(. \mid x)|$ is continuous and ultimately decreasing to 0 . (A1) implies that the conditional distribution of $Y$ given $X=x$ is in the Fréchet maximum domain of attraction. In this context, $\gamma(x)$ is referred to as the conditional tail-index since it tunes the tail heaviness of the conditional distribution of $Y$ given $X=x$. Assumption (A1) also yields that $\bar{F}(. \mid x)$ is regularly varying at infinity with index $-1 / \gamma(x)$. i.e for all $\zeta>0$,

$$
\lim _{y \rightarrow \infty} \frac{\bar{F}(\zeta y \mid x)}{\bar{F}(y \mid x)}=\zeta^{-1 / \gamma(x)}
$$

The function $\varepsilon(. \mid x)$ plays an important role in extreme-value theory since it drives the speed of convergence in (3) and more generally the bias of extreme-value estimators. 
Therefore, it may be of interest to specify how it converges to 0. In (Gomes et al., 2000), the auxiliary function is supposed to be regularly varying and the estimation of the corresponding regular variation index is addressed. Some Lipschitz conditions are also required:

(A2): There exist $\kappa_{\varepsilon}, \kappa_{c}, \kappa_{\gamma}>0$ and $u_{0}>1$ such that for all $\left(x, x^{\prime}\right) \in E^{2}$ and $u>u_{0}$,

$$
\begin{aligned}
\left|\log c(x)-\log c\left(x^{\prime}\right)\right| & \leq \kappa_{c} d\left(x, x^{\prime}\right), \\
\left|\varepsilon(u \mid x)-\varepsilon\left(u \mid x^{\prime}\right)\right| & \leq \kappa_{\varepsilon} d\left(x, x^{\prime}\right), \\
\left|1 / \gamma(x)-1 / \gamma\left(x^{\prime}\right)\right| & \leq \kappa_{\gamma} d\left(x, x^{\prime}\right) .
\end{aligned}
$$

The last assumptions are standard in the kernel estimation framework.

(A3): $K$ is a function with support $[0,1]$ and there exist $0<C_{1}<C_{2}<\infty$ such that $C_{1} \leq K(t) \leq C_{2}$ for all $t \in[0,1]$.

(A4): $q$ is a probability density function (pdf) with support $[-1,1]$.

One may also assume without loss of generality that $K$ integrates to one. In this case, $K$ is called a type I kernel, see (Ferraty and Vieu, 2006, Definition 4.1). Finally, let $B(x, h)$ be the ball of center $x$ and radius $h$. The small ball probability of $X$ is defined by $\varphi_{x}(h)=\mathbb{P}(X \in B(x, h))$. Under (A3), for all $\tau>0$, the $\tau$ th moment is defined by $\mu_{x}^{(\tau)}(h)=\mathbb{E}\left\{K^{\tau}(d(x, X) / h)\right\}$.

\section{Main results}

Let us first focus on the estimation of small tail probabilities $\bar{F}\left(y_{n} \mid x\right)$ when $y_{n} \rightarrow \infty$ as $n \rightarrow \infty$. The following result provides sufficient conditions for the asymptotic normality of $\hat{\bar{F}}_{n}\left(y_{n} \mid x\right)$.

Theorem 1. Suppose (A1) - (A4) hold. Let $x \in E$ such that $\varphi_{x}(h)>0$ and introduce $y_{n, j}=a_{j} y_{n}$ for $j=1, \ldots, J$ with $0<a_{1}<a_{2}<\ldots<a_{J}$ and where $J$ is a positive integer. If $y_{n} \rightarrow \infty$ such that $n \varphi_{x}(h) \bar{F}\left(y_{n} \mid x\right) \rightarrow \infty, n \varphi_{x}(h) \bar{F}\left(y_{n} \mid x\right)\left(\lambda / y_{n}\right)^{2} \rightarrow 0$ and $n \varphi_{x}(h) \bar{F}\left(y_{n} \mid x\right)\left(h \log y_{n}\right)^{2} \rightarrow 0$ as $n \rightarrow \infty$, then

$$
\left\{\sqrt{n \mu_{x}^{(1)}(h) \bar{F}\left(y_{n} \mid x\right)}\left(\frac{\hat{\bar{F}}_{n}\left(y_{n, j} \mid x\right)}{\bar{F}\left(y_{n, j} \mid x\right)}-1\right)\right\}_{j=1, \ldots, J}
$$

is asymptotically Gaussian, centered, with covariance matrix $C(x)$ where $C_{j, j^{\prime}}(x)=$ $a_{j \wedge j^{\prime}}^{1 / \gamma(x)}$ for $\left(j, j^{\prime}\right) \in\{1, \ldots, J\}^{2}$.

Note that $n \varphi_{x}(h) \bar{F}\left(y_{n} \mid x\right) \rightarrow \infty$ is a necessary and sufficient condition for the almost sure presence of at least one sample point in the region $B(x, h) \times\left(y_{n}, \infty\right)$ of $E \times \mathbb{R}$. Thus, this natural condition states that one cannot estimate small tail probabilities out of the sample using $\hat{\bar{F}}_{n}$. This result may be compared to (Einmahl, 1990) which establishes the asymptotic behavior of the empirical survival function in the unconditional case but 
without assumption on the distribution. Letting $\sigma_{n}(x)=\left(n \mu_{x}^{(1)}(h) \alpha_{n}\right)^{-1 / 2}$, the asymptotic normality of $\hat{q}_{n}\left(\alpha_{n} \mid x\right)$ when $\alpha_{n} \rightarrow 0$ as $n \rightarrow \infty$ can be established under similar conditions.

Theorem 2. Suppose (A1) - (A4) hold. Let $x \in E$ such that $\varphi_{x}(h)>0$ and introduce $\alpha_{n, j}=\tau_{j} \alpha_{n}$ for $j=1, \ldots, J$ with $\tau_{1}>\tau_{2}>\ldots>\tau_{J}>0$ and where $J$ is a positive integer. If $\alpha_{n} \rightarrow 0$ such that $\sigma_{n}(x) \rightarrow 0, \sigma_{n}^{-1}(x) \lambda / q\left(\alpha_{n} \mid x\right) \rightarrow 0$ and $\sigma_{n}^{-1}(x) h \log \alpha_{n} \rightarrow 0$ as $n \rightarrow \infty$, then

$$
\left\{\sigma_{n}^{-1}(x)\left(\frac{\hat{q}_{n}\left(\alpha_{n, j} \mid x\right)}{q\left(\alpha_{n, j} \mid x\right)}-1\right)\right\}_{j=1, \ldots, J}
$$

is asymptotically Gaussian, centered, with covariance matrix $\gamma^{2}(x) \Sigma$ where $\Sigma_{j, j^{\prime}}=1 / \tau_{j \wedge j^{\prime}}$ for $\left(j, j^{\prime}\right) \in\{1, \ldots, J\}^{2}$.

The functional kernel estimator of extreme quantiles $\hat{q}_{n}\left(\alpha_{n} \mid x\right)$ requires a stringent condition on the order $\alpha_{n}$ of the quantile, since by construction it cannot extrapolate beyond the maximum observation in the ball $B(x, h)$. To overcome this limitation, a Weissman type estimator (Weissman, 1978) can be derived:

$$
\hat{q}_{n}^{\mathrm{W}}\left(\beta_{n} \mid x\right)=\hat{q}_{n}\left(\alpha_{n} \mid x\right)\left(\alpha_{n} / \beta_{n}\right)^{\hat{\gamma}_{n}(x)} .
$$

Here, $\hat{q}_{n}\left(\alpha_{n} \mid x\right)$ is the functional kernel estimator of the extreme quantile considered so far and $\hat{\gamma}_{n}(x)$ is a functional estimator of the conditional tail-index $\gamma(x)$. As illustrated in the next theorem, the extrapolation factor $\left(\alpha_{n} / \beta_{n}\right)^{\hat{\gamma}_{n}(x)}$ allows to estimate extreme quantiles of order $\beta_{n}$ arbitrary small.

Theorem 3. Suppose (A1)-(A4) hold. Let us introduce

- $\alpha_{n} \rightarrow 0$ such that $\sigma_{n}(x) \rightarrow 0, \sigma_{n}^{-1}(x) \lambda / y_{n} \rightarrow 0$ and $\sigma_{n}^{-1}(x) h \log \alpha_{n} \rightarrow 0$ as $n \rightarrow \infty$,

- $\left(\beta_{n}\right)$ such that $\beta_{n} / \alpha_{n} \rightarrow 0$ as $n \rightarrow \infty$,

- $\hat{\gamma}_{n}(x)$ such that $\sigma_{n}^{-1}(x)\left(\hat{\gamma}_{n}(x)-\gamma(x)\right) \stackrel{d}{\longrightarrow} \mathcal{N}\left(0, v^{2}(x)\right)$ where $v^{2}(x)>0$.

Then, for all $x \in E$,

$$
\frac{\sigma_{n}^{-1}(x)}{\log \left(\alpha_{n} / \beta_{n}\right)}\left(\frac{\hat{q}_{n}^{W}\left(\beta_{n} \mid x\right)}{q\left(\beta_{n} \mid x\right)}-1\right) \stackrel{d}{\longrightarrow} \mathcal{N}\left(0, v^{2}(x)\right) .
$$

Note that, when $K$ is the pdf of the uniform distribution, this result is consistent with (Gardes et al., 2010, Theorem 3), obtained in a fixed-design setting.

Let us now give some examples of functional estimators of the conditional tail-index. Let $\alpha_{n} \rightarrow 0$ and $\tau_{1}>\tau_{2}>\ldots>\tau_{J}>0$ where $J$ is a positive integer. Two additionary notations are introduced for the sake of simplicity: $u=(1, \ldots, 1)^{t} \in \mathbb{R}^{J}$ and $v=$ $\left(\log \left(1 / \tau_{1}\right), \ldots, \log \left(1 / \tau_{J}\right)\right)^{t} \in \mathbb{R}^{J}$. The following family of estimators is proposed

$$
\hat{\gamma}_{n}(x)=\frac{\varphi\left(\log \hat{q}_{n}\left(\tau_{1} \alpha_{n} \mid x\right), \ldots, \log \hat{q}_{n}\left(\tau_{J} \alpha_{n} \mid x\right)\right)}{\varphi\left(\log \left(1 / \tau_{1}\right), \ldots, \log \left(1 / \tau_{J}\right)\right)},
$$


where $\varphi: \mathbb{R}^{J} \rightarrow \mathbb{R}$ denotes a twice differentiable function verifying the shift and location invariance conditions $\varphi(\theta v)=\theta \varphi(v)$ for all $\theta>0$ and $\varphi(\eta u+x)=\varphi(x)$ for all $\eta \in \mathbb{R}$ and $x \in \mathbb{R}^{J}$. For instance, introducing the auxiliary function $m_{p}\left(x_{1}, \ldots, x_{J}\right)=\sum_{j=1}^{J}\left(x_{j}-\right.$ $\left.x_{1}\right)^{p}$ for all $p>0$ and considering $\varphi_{\mathrm{H}}(x)=m_{1}(x)$ gives rise to a kernel version of the Hill estimator (Hill, 1975):

$$
\hat{\gamma}_{n}^{\mathrm{H}}(x)=\sum_{j=1}^{J}\left[\log \hat{q}_{n}\left(\tau_{j} \alpha_{n} \mid x\right)-\log \hat{q}_{n}\left(\alpha_{n} \mid x\right)\right] / \sum_{j=1}^{J} \log \left(1 / \tau_{j}\right) .
$$

Generalizations of the kernel Hill estimator can be obtained with $\varphi(x)=m_{p}(x) / m_{1}^{p-1}(x)$, see (Gomes and Martins, 2001, equation (2.2)), $\varphi(x)=m_{p}^{1 / p}(x)$, see e.g. (Segers, 2001, example (a)) or $\varphi(x)=m_{p \theta}^{1 / \theta}(x) / m_{p-1}(x), p \geq 1, \theta>0$, see (Caeiro and Gomes, 2002). In the case where $J=3, \tau_{1}=4, \tau_{2}=2$ and $\tau_{3}=1$, the function

$$
\varphi_{\mathrm{P}}\left(x_{1}, x_{2}, x_{3}\right)=\log \left(\frac{\exp x_{2}-\exp x_{1}}{\exp x_{3}-\exp x_{2}}\right)
$$

leads us to a kernel version of Pickands estimator (Pickands, 1975)

$$
\hat{\gamma}_{n}^{\mathrm{P}}(x)=\frac{1}{\log 2} \log \left(\frac{\hat{q}_{n}\left(\alpha_{n} \mid x\right)-\hat{q}_{n}\left(2 \alpha_{n} \mid x\right)}{\hat{q}_{n}\left(2 \alpha_{n} \mid x\right)-\hat{q}_{n}\left(4 \alpha_{n} \mid x\right)}\right) .
$$

We refer to (Gijbels and Peng, 2000) for a different variant of Pickands estimator in the context where the distribution of $Y$ given $X=x$ has a finite endpoint. The asymptotic normality of $\hat{\gamma}_{n}(x)$ is a consequence of Theorem 2 .

Theorem 4. Under assumptions of Theorem 2 and if $\sigma_{n}^{-1}(x) \varepsilon\left(q\left(\tau_{1} \alpha_{n} \mid x\right) \mid x\right) \rightarrow 0$ as $n \rightarrow \infty$, then, $\sigma_{n}^{-1}(x)\left(\hat{\gamma}_{n}(x)-\gamma(x)\right)$ converges to a centered Gaussian random variable with variance

$$
V(x)=\frac{\gamma^{2}(x)}{\varphi^{2}(v)}(\nabla \varphi(\gamma(x) v))^{t} \Sigma(\nabla \varphi(\gamma(x) v)) .
$$

As an illustration, in the case of the kernel Hill and Pickands estimators, we obtain

$$
\begin{aligned}
V_{\mathrm{H}}(x) & =\gamma^{2}(x)\left(\sum_{j=1}^{J} \frac{2(J-j)+1}{\tau_{j}}-J^{2}\right) /\left(\sum_{j=1}^{J} \log \left(1 / \tau_{j}\right)\right)^{2} . \\
V_{\mathrm{P}}(x) & =\frac{\gamma^{2}(x)\left(2^{2 \gamma(x)+1}+1\right)}{4(\log 2)^{2}\left(2^{\gamma(x)}-1\right)^{2}} .
\end{aligned}
$$

Clearly, $V_{\mathrm{P}}(x)$ is the variance of the classical Pickands estimator, see for instance (de Haan and Ferreira, 2006, Theorem 3.3.5). Focusing on the kernel Hill estimator and choosing $\tau_{j}=1 / j$ for each $j=1, \ldots, J$ yields $V_{\mathrm{H}}(x)=\gamma^{2}(x) J(J-1)(2 J-1) /\left(6 \log ^{2}(J !)\right)$. In this case, $V_{\mathrm{H}}(x)$ is a convex function of $J$ and is minimum for $J=9$ leading to $V_{\mathrm{H}}(x) \simeq 1.25 \gamma^{2}(x)$. 


\section{References}

A. Berlinet, A. Gannoun and E. Matzner-Løber. Asymptotic normality of convergent estimates of conditional quantiles. Statistics, 35:139-169, 2001.

F. Caeiro and M.I. Gomes. Bias reduction in the estimation of parameters of rare events. Theory of Stochastic Processes, 8:67-76, 2002.

J.H.J Einmahl. The empirical distribution function as a tail estimator, Statistica Neerlandica, 44:79-82, 1990.

F. Ferraty and P. Vieu. Nonparametric functional data analysis. Springer, 2006.

F. Ferraty, A. Rabhi and P. Vieu. Conditional quantiles for dependent functional data with application to the climatic El Nino Phenomenon, Sankhya: The Indian Journal of Statistics, 67(2):378-398, 2005.

A. Gannoun. Estimation non paramétrique de la médiane conditionnelle, médianogramme et méthode du noyau, Publications de l'Institut de Statistique de l'Université de Paris, XXXXVI:11-22, 1990.

L. Gardes, S. Girard and A. Lekina. Functional nonparametric estimation of conditional extreme quantiles. Journal of Multivariate Analysis, 101:419-433, 2010.

I. Gijbels and L. Peng. Estimation of a support curve via order statistics. Extremes, $3: 251-277,2000$.

M.I. Gomes and M.J. Martins and M. Neves. Semi-parametric estimation of the second order parameter, asymptotic and finite sample behaviour. Extremes, 3:207-229, 2000.

M.I. Gomes and M.J. Martins. Generalizations of the Hill estimator - asymptotic versus finite sample behaviour. Journal of Statistical Planning and Inference, 93:161-180, 2001. L. de Haan and A. Ferreira. Extreme Value Theory: An Introduction, Springer Series in Operations Research and Financial Engineering, Springer, 2006.

B.M. Hill. A simple general approach to inference about the tail of a distribution. The Annals of Statistics, 3:1163-1174, 1975.

J. Pickands. Statistical inference using extreme order statistics. The Annals of Statistics, 3:119-131, 1975.

G.G. Roussas. Nonparametric estimation of the transition distribution function of a Markov process. Ann. Math. Statist., 40:1386-1400, 1969.

T. Samanta. Non-parametric estimation of conditional quantiles. Statistics and Probability Letters, 7:407-412, 1989.

J. Segers. Residual estimators. Journal of Statistical Planning and Inference, 98:15-27, 2001.

C.J. Stone. Consistent nonparametric regression (with discussion). The Annals of Statistics, 5:595-645, 1977.

W. Stute. Conditional empirical processes. The Annals of Statistics, 14:638-647, 1986.

I. Weissman. Estimation of parameters and large quantiles based on the $k$ largest observations, Journal of the American Statistical Association, 73:812-815, 1978. 